\section{Color selectivity in motion aftereffect}

\author{
W. J. LOVEGROVE \\ University of Tasmania, Hobart, Australia \\ RAY OVER \\ La Trobe University, Bundoora, Australia
}

and

\section{JACK BROERSE \\ Mount Gravatt College, Brisbane, Australia}

In the motion aftereffect, stationary lines seem to be moving in the direction opposite to previously inspected moving lines. Lovegrove, Over, and Broerse (1972) found that this aftereffect is reduced in strength when the inspection and test stimuli are displayed in different colored light. Mayhew (1975) replicated this finding, and other studies (Favreau, 1976; Favreau, Emerson, \& Corballis, 1972; Mayhew, 1972, 1975; Mayhew \& Anstis, 1972; Murch, 1974), employing variants of the classical aftereffect paradigm, have yielded uniform evidence that the motion aftereffect is color selective. Day and Wade (1979), in treating Lovegrove et al.'s results as an anomalous finding, have disregarded these studies; instead, they support their own demonstrations that the motion aftereffect lacks color specificity by reference to Anstis (1964) and Wohlgemuth (1911). The present paper, as well as reviewing experimental evidence relevant to the claim that at least some motion detectors in human vision are color coded, suggests a basis for the discrepant results obtained by Day and Wade (1979) and Lovegrove et al. (1972).

Two experimental paradigms have been used to demonstrate color selectivity in the motion aftereffect. In one (contingent aftereffect), there is alternate display of opposite color-motion combinations before the subject reports the perceived motion of a stationary pattern presented under various chromatic conditions, and in the other (simple aftereffect), the perceived motion of the colored test pattern is measured following exposure to a single color-motion combination. Investigators using both paradigms have generally assumed that distortion in motion perception results to the extent that detectors adapted as a consequence of inspection would normally have been engaged in representation of the test stimulus. The contingent aftereffect paradigm (Favreau, 1976; Favreau et al., 1972; Mayhew, 1972, 1975; Mayhew \& Anstis, 1972; Murch, 1974) factors out the influence of color-insensitive motion detectors. The method requires subjects to view, in alternation, clockwise rotation of a spiral in red light and counterclockwise rotation of the spiral in green light; the subjects subsequently report that a stationary spiral seems to be rotating slightly in the counterclockwise direction when seen in red light and in the clockwise direction when viewed in green light. These aftereffects cannot be due to differential adaptation of color-insensitive motion detectors, since alternate exposure to clockwise and counterclockwise rotation of a spiral in white light does not yield directional aftereffects (Wohlgemuth, 1911). Alternate exposure to opposite color-spatial combinations would, however, produce differential adaptation of color-motion detectors; for example, following clockwise rotation in red light and counterclockwise rotation in green light, clockwise-red detectors would be adapted relative to counterclockwise-red, as would counterclockwisegreen relative to clockwise-green.

The simple motion aftereffect has been explained by assuming that normally stationary lines are signaled by a balance in activity between motiondetecting systems with opposed directional selectivity, but this balance is upset when the system excited by presentation of lines moving in one direction is subsequently suppressed. Lovegrove et al. (1972) supposed that the motion aftereffect was reduced when there was a color shift from inspection to test stages because this condition yields selective adaptation of the color-insensitive, but not of the colorsensitive, motion detectors by which the test stimulus would normally be represented. Both their results and those later obtained by Mayhew (1975) using the same paradigm are consistent with this explanation. In contrast, Day and Wade (1979) have found that the strength of the motion aftereffect is independent of the color relationship between the inspection and test patterns. Day and Wade have claimed that their results are consistent with "the weight of evidence" [in this respect, they cite Anstis (1964) and Wohlgemuth (1911), but not the other literature reviewed above], while those of Lovegrove et al. are "the solitary discrepancy." Despite the implication by Day and Wade to the contrary, Wohlgemuth (1911) did not measure the motion aftereffect when the stationary test pattern was of a different color from that of the moving inspection pattern. In addition, Anstis (1964) did not investigate whether the motion aftereffect in photopic vision changes in magnitude when the inspection and test patterns differ in color. His experiment, concerned with transfer of the motion aftereffect between photopic and scotopic vision, used red and blue filters to segregate inputs to cone and rod systems, respectively. The stimuli were observed in parafoveal and peripheral vision by darkadapted subjects, and the transfer of aftereffects 
between rod and cone systems may merely have involved color-insensitive motion detectors.

Two differences in experimental procedure between Day and Wade (1979) and Lovegrove et al. (1972) may be basic to the discrepant results that were obtained. First, color-selective effects may have been neutralized in the two studies in which Day and Wade measured the spiral aftereffect, since subjects were exposed to opposite colors during inspection within the same session. Second, in Experiment 3 (which replicated in other respects the conditions employed by Lovegrove et al..), Day and Wade, rather than using the same grating as the inspection and test stimulus, required subjects to inspect the moving grating in one spatial location and the test grating in a different location. This difference, which in itself is not important, meant that test judgments commenced immediately upon cessation of inspection (Day, Note 1), whereas the experimental arrangement employed by Lovegrove et al. imposed an interstimulus interval of $10 \mathrm{sec}$ on a color change between inspection and test stages. The same interval was maintained when the color was identical in the inspection and test stages of the experiment. Interstimulus interval would be an important variable if the motion aftereffect has short-term components that lack color tuning, and longer-term components that exhibit selectivity to the color relationship between inspection and test patterns. There is some evidence that such may be the case.

Bonnet and Pouthas (1972) and Favreau (1976) have demonstrated two phases in decay of the motion aftereffect. Bonnet and Pouthas found that fast, vivid movement was visible on a noise background immediately following inspection of moving lines, but thereafter the aftereffect took the form of slower and less vivid movement that was seen best when the inspection and test stimuli were similar. In the first stage reported by Favreau, the motion aftereffect showed rapid decay and it was sensitive to brightnesscontrast but not to color, while in the second stage, the aftereffect decayed slowly (it, in fact, increased in strength over time under some conditions) and was color selective. The two phases of the motion aftereffect may reflect the differential sensitivity of transient and sustained systems in visual processing. There is both psychophysical and electrophysiological evidence that transient detectors are relatively insensitive to spatial detail and lacking in color selectivity, whereas sustained detectors exhibit spatial and color tuning (Dow \& Gouras, 1973; Gouras, 1974; Tolhurst, 1977).

From the above analysis, the interstimulus interval between inspection and test stages might be an important determinant of whether color-selectivity is obtained with the simple motion aftereffect. One of us (W. J. Lovegrove) is currently testing this possibility, which may be basic to the discrepancy between results obtained by Day and Wade (1979) and Lovegrove et al. (1972). Mayhew (1975) was able to demonstrate color selectivity in the simple motion aftereffect using a short interstimulus interval. However, the components of the inspection stimulus used by Mayhew differed in color but not in brightness, and an initial component of the aftereffect reflecting adaptation of transient detectors would thus not be obtained. Favreau (1976) similarly found that the contingent motion aftereffect was color selective immediately following exposure to a moving display that contained color-contrast but not brightnesscontrast information. When the components of the inspection stimulus differed in brightness and color, it was not until some time after the inspection period that the motion aftereffect exhibited color selectivity.

Simultaneous motion-contrast studies have provided further evidence that at least some motion detectors in human vision are color coded. Stationary lines appear to move upward either when viewed following exposure to downward moving lines (aftereffect) or when surrounded by downward moving lines (simultaneous contrast). This latter distortion has been attributed to inhibitory interaction between directionally sensitive motion detectors. Over and Lovegrove (1973) suggested that these mechanisms might be color tuned, since simultaneous motion contrast is reduced when the center and the surround gratings differ in color. Day and Dickinson (1977) replicated Over and Lovegrove's results using a similar display, although they found that Dunckertype induced motion was insensitive to the color relationship between juxtaposed stationary and moving components.

Lastly, in suggesting that a distinction needed to be made between simple and contingent aftereffects, Day and Wade (1979) implied that the perceived motion of a stationary test pattern might be selective to color following alternate exposure to opposite color-motion combinations, but not following exposure to lines moving in one direction in a single color. This proposition, which Day and Wade did not test in their experiments, relied upon the demonstration by Stromeyer and Mansfield (1970) that stimulus alternation during inspection is a necessary condition for induction of motion-specific color aftereffects. In using this result to formulate a general principle, Day and Wade have ignored evidence that aftereffects demonstrating color-motion (Mayhew, 1975), color-orientation (May, 1972; Piggins \& Leppmann, 1973; Stromeyer, 1972), and color-spatial frequency (May, 1972; Stromeyer, 1972) specificity have been induced by exposure to a single color-spatial combination. The colororientation selectivity found in the visual evoked potential by May, Leftwich, and Aptaker (1974) was 
also produced by adaptation to a single colored grating. Further, simple and contingent color-selective motion aftereffects do not have independent bases, since prior exposure to opposite color-motion combinations shifts the distortion that is subsequently induced by inspection of a single color-motion value (Mayhew, 1975). An adequate explanation of colorspatial aftereffects must thus encompass effects induced by inspection of both single and double color-spatial combinations.

\section{REFERENCE NOTE}

1. Day, R. H. Personal communication, June 1978.

\section{REFERENCES}

Anstis, S. M. After-effect of seen motion: Transfer from rods to cones and vice versa. Nature, 1964, 201, 952.

Bonnet, C., \& Pouthas, V. Interactions between spatial and kinetic dimensions in movement aftereffect. Perception \& Psychophysics, 1972, 12, 193-200.

DAY, R. H., \& Dickinson, R. G. Absence of color-selectivity in Duncker-type induced visual movement. Perception \& Psychophysics, 1977, 22, 313-320.

DAY, R. G., \& WADE, N. Absence of color sensitivity in the visual motion aftereffect. Perception \& Psychophysics, 1979, 25, 00-00.

Dow, B. M., \& Gouras, P. Color and spatial specificity of single units in rhesus monkey foveal striate cortex. Journal of Neurophysiology, 1973, 36, 79-100.

FaVREAU, O. E. Interference in color-contingent motion aftereffects. Quarterly Journal of Experimental Psychology, 1976, 28, 553-560.

Favreau, O., Emerson, V., \& Corballis, M. Motion perception: A color-contingent aftereffect. Science, 1972, 176, 78-79.
Gouras, P. Opponent-colour cells in different layers of foveal striate cortex. Journal of Physiology, 1974, 238, 583-602.

Lovegrove, W. J., Over, R., \& BROERse, J. Colour selectivity in motion after-effect. Nature, 1972, 238, 334-335.

MAY, J. G. Chromatic adaptation of orientation- and size-specific visual processes in man. Vision Research, 1972, 12, 1509-1517.

May, J. G., LefTwich, D. A., \& APTAKER, P. Evoked potential correlates of adaptation to wavelength and orientation. Vision Research, 1974, 14, 143-146.

MAYHEW, J. E. W. Directional asymmetry in the duration of simple motion aftereffects produced by movement aftereffects contingent on colour. Perception, 1972, 1, 453-457.

MAYHEW, J. E. W. The effect of stationary patterns on adaptation for movement: Evidence for inhibitory interaction. Perception, $1975,4,311-329$.

Ma Yhew, J. E. W., \& Anstis, S. M. Movement aftereffects contingent on color, intensity and pattern. Perception \& Psychophysics, 1972, 12, 77-85.

MURCH, G. M. Color contingent motion aftereffects: Single or multiple levels of processing. Vision Research, 1974, 14, 1181-1184.

OVER, R., \& Lovegrove, W. J. Color selectivity in simultaneous motion contrast. Perception \& Psychophysics, 1973, 14, 445-448.

Piggins, D. J., \& Leppmann, P. K. Role of retinal image in evoking the McCollough effect. Nature New Biology, 1973, 245, 255-256.

STROME YER, C. F. Edge-contingent color after-effects: Spatial frequency selectivity. Vision Research, 1972, 12, 717-733.

Strome YeR, C. F., \& Mansfield, R. Colored aftereffects produced with moving edges. Perception \& Psychophysics, 1970, 7, 108-114.

TolfuRst, D. J. Colour-coding properties of sustained and transient channels in human vision. Nature, 1977, 266, 266-267.

WOHLGEMUTH, A. On the after-effect of seen motion. British Journal of Psychology Monograph Supplement, 1911, 1, 1-117.

(Received for publication November 14, 1978; accepted November 14, 1978.) 